\title{
Equilibrio líquido-vapor para el sistema cuaternario Agua-Acetona-Butanol-Etanol a Presiones Bajas
}

\section{Liquid-vapor equilibrium for the Water-Acetone-Butanol- Ethanol quaternary system at Low Pressures}

\author{
Jairo Antonio Cubillos Lobo ${ }^{a}$ \\ Jorge Arturo Moreno Cascante ${ }^{b}$
}

Fecha de Recepción: 09.06.2018

Fecha de Aceptación: 10.10.2018

DOI: https://doi.org/10.19053/01217488.v10.n1.2019.8063

\begin{abstract}
Resumen
Debido a la existencia de un comportamiento o interacción no ideal del etanol, butanol y acetona en soluciones acuosas, se presenta en este trabajo un método para predecir aquellas propiedades en mezcla que dependen de la composición, temperatura y presión, especialmente la actividad y la fugacidad. Estas propiedades son importantes para estimar constantes de equilibrio, constantes de velocidad de transferencia y reacción química, dimensionamiento y diseño de equipos de proceso. Actualmente, el biobutanol se considera un sustituto del bioetanol como biocombustible al presentar ventajas como mayor capacidad calorífica y menor presión de vapor. Su obtención se realiza a partir de la fermentación Acetona-ButanolEtanol (ABE), donde sus productos mayoritarios son acetona, butanol y etanol en una relación molar 3:6:1, respectivamente. Por ello, se calculó el equilibrio líquido-vapor para el sistema cuaternario agua-acetonabutanol-etanol a presiones reducidas de $0.1,0.5$ y $1 \mathrm{kPa}$, con el propósito de evaluar el comportamiento de los azeótropos (agua-butanol y agua-etanol), los cuales como bien se conocen límitan la aplicación de un proceso de separación. Se utilizó el modelo Non Random Two Liquid (NRTL) para calcular el coeficiente de actividad de los componentes en fase líquida, las ecuaciones del virial para calcular el coeficiente de fugacidad de los componentes en fase de vapor y el algoritmo Rachford y Rice para el punto de roció. De esta manera, se pudo demostrar que a presiones bajas el azeótropo que se forma entre agua y butanol desaparece paulatinamente mientras se reduce la presión. Por tanto, se recomienda utilizar una presión de $0.1 \mathrm{kPa}$ para la destilación al vacío y $0,5 \mathrm{kPa}$ para la pervaporacion. Estos valores están condicionados a la estabilidad del medio de separación (membrana en el caso de la pervaporación) y a la evaluación económica del proceso.
\end{abstract}

Palabras clave: Butanol, actividad, fugacidad, azeótropo, sistema cuaternario.

a b Universidad Pedagógica y Tecnológica de Colombia. 


\begin{abstract}
Due to the ocurrence of a non ideal behavior of ethanol, butanol and acetone in aqueous solutions, a method to predict those properties in mixture that depend on the composition, temperature and pressure, especially the activity and the fugacity, is outlined in this work. These properties are very important for estimating equilibrium constants, constants of transfer speed and chemical reaction, sizing and design of process equipment. Currently, biobutanol is considered a substitute for bioethanol as a biofuel, presenting advantages, such as: higher heat capacity and lower vapor pressure. It is obtained from the fermentation of Acetone-Butanol-Ethanol (ABE), where its major products are acetone, butanol and ethanol in a molar ratio of 3: 6: 1, respectively. Therefore, the liquid-vapor equilibrium for the quaternary system water-acetonebutanol-ethanol was calculated at reduced pressures of $0.1,0.5$ and $1 \mathrm{kPa}$, with the purpose of evaluating the behavior of the azeotropes (water-butanol and water-ethanol), which is a key issue in the application of a separation process. The Non Random Two Liquid model (NRTL) was used to calculate the activity coefficient of the components in the liquid phase, the virial equations to calculate the fugacity coefficient of the components in the vapor phase and the Rachford and Rice algorithm for the dew temperature. In this way, it could be demonstrated that at low pressures the azeotrope formed between water and butanol slighly disappears while the pressure is going down. Therfore, it is recommended by using a pressure of $0.1 \mathrm{kPa}$ for vacuum distillation, while $0.5 \mathrm{kPa}$ for pervaportation. These values depend on the separation system stability (membranes in case of pervaporation) and the process economical balance.
\end{abstract}

Keywords: Butanol, activity, fugacity, azeotrope, quaternary system.

\section{INTRODUCCIÓN}

Actualmente, el butanol es un compuesto de gran interés en la industria química, debido a sus múltiples aplicaciones. Entre estas se destaca su uso como biocombustible alternativo al etanol dado su mayor contenido energético (2,510.2 $\mathrm{kJ} / \mathrm{mol}$ para el butanol y $1,278.6 \mathrm{~kJ} / \mathrm{mol}$ para el etanol); menor calor de vaporización $(7.85 \mathrm{~kJ} / \mathrm{mol}$ para el butanol y $19.62 \mathrm{~kJ} / \mathrm{mol}$ para el etanol) lo que hace que el butanol presente menos pérdidas por evaporación al ambiente[1][2], mayor solubilidad en la gasolina y diésel debido a sus dos grupos metilenos adicionales, lo que hace al butanol más apolar y por ello más afin con estos combustibles [3] y por último el butanol presenta mayor viscosidad que el etanol (2.544 $\mathrm{mPa}$ para el butanol y $1.088 \mathrm{mPa}$ para el etanol), lo que representa un menor desgaste de los pistones y por consiguiente aumenta la vida útil del motor. Igualmente, debido a su alta solubilidad en disolventes orgánicos (cetonas, aldehídos, ésteres e hidrocarburos alifáticos y aromáticos), el butanol es ampliamente utilizado en el sector de pinturas, farmacéutico, automotriz, textil, entre otros.

Desde mediados del siglo XIX, el butanol se produce a través de procesos petroquímicos, sin embargo, existe interés en nuevas tecnologías limpias que usen recursos renovables, como es caso de un proceso biológico a base de microorganismos (Clostridium anaerobios) [4].
En los procesos fermentativos se obtienen otros metabólicos diferentes al butanol, tales como: acetona y etanol (heterofermentación) [5]; por tanto, el caldo de fermentación queda compuesto principalmente por acetona, butanol, etanol y agua. Debido a que el butanol no puede separarse de esta mezcla con métodos de separación físicomecánicos [6] comúnmente se emplean diferentes operaciones unitarias, tales como: destilación, pervaporación, absorción y extracción líquidolíquido [7] [8].

Para el diseño de las operaciones unitarias donde hay cambio de fase de líquido a vapor, como son la pervaporación y la destilación, se requiere el cálculo de propiedades de mezcla y la simulación del equilibrio líquido-vapor [9]. En este trabajo se calcularon los coeficientes de actividad en la fase líquida y los coeficientes de fugacidad en la fase vapor, que son datos fundamentales para la modelación del equilibrio líquido-vapor, el diseño de equipos de separación y el establecimiento de las condiciones óptimas de operación. En particular, se reporta una forma para modelar el equilibrio líquido-vapor de un sistema no ideal de cuatro componentes acetonabutanol-etanol-agua. Esta mezcla presenta azeótropos entre los pares de componentes AguaButanol y Agua-Etanol [10].

La simulación se realizó a presiones bajas $(0.1,0.5$ y $1 \mathrm{kPa})$, debido a que se pretende 
evaluar el comportamiento que presentan los azeótropos y para determinar si son factibles procesos de separación como la destilación al vacío y la pervaporación. Se utilizó el modelo NRTL para calcular el coeficiente de actividad de los componentes en fase líquida, mientras que el modelo del Virial se utilizó para calcular el coeficiente de fugacidad para los componentes en fase vapor junto con el algoritmo Rachford y Rice para la determinación de los puntos de rocío.

\section{METODOLOGÍA}

Para el desarrollo de este trabajo se utilizó el modelo NRTL para calcular el coeficiente de actividad de los componentes i en la mezcla líquida, el modelo del Virial para calcular el coeficiente de fugacidad para los componentes $i$ en la fase vapor y el algoritmo de Rachford y Rice para la estimación del punto de rocío. La simulación se realizó a presión constante y para este caso se eligieron 4 presiones diferentes. Así, 3 de estos se realizaron a presión de vacío que sirven para el funcionamiento de un sistema de pervaporación y destilación al vacío $(0.1,0.5$, $1 \mathrm{kPa})$; la cuarta presión usada se realizó a una presión cercana al ambiente $(100 \mathrm{kPa})$ para observar el comportamiento de este sistema a medida que disminuye la presión; además para cada presión se establecieron 4 concentraciones de etanol y acetona $(2 \%, 5 \%, 10 \%$ y $15 \% p / p)$.

Se utilizó la extensión Macros del software Microsoft Excel para programar el modelo de actividad, el modelo de fugacidad y el algoritmo para el equilibrio líquido-vapor.

\subsection{Modelo de NRTL}

La aplicación del modelo NRTL es útil para estimar el comportamiento de soluciones completamente miscibles, mezcla de hidrocarburos y alcoholes. Los parámetros binarios que maneja este modelo depende de la temperatura, haciéndolo un modelo flexible y utilizable en un rango amplio [11]. El modelo NRTL surge a partir del modelo de Wilson y se combina con las ventajas de la ecuación de Van Laar, el cual es un modelo muy útil para equilibrios de fases L-V, L-L y L-L-V y se basa en el concepto de celdas líquidas y mecánica estadística para representar la estructura líquida [10]. El modelo NRTL viene dado por la ecuación 1[12]:

$$
L n \gamma_{i}=\frac{\sum_{j=1}^{n} x_{j} T_{j i} G_{j i}}{\sum_{K=1}^{n} x_{K} G_{K i}}+\sum_{j=1}^{n} \frac{x_{j} G_{i j}}{\sum_{K=1}^{n} x_{K} G_{K j}} T_{i j}-\frac{\sum_{m=1}^{n} x_{m} T_{m j} G_{m j}}{\sum_{K=1}^{n} x_{K} G_{K j}}
$$

Donde $\gamma_{i}$ es la actividad del componente $i$ en la mezcla líquida y $x_{i}$ es la fracción molar del componente $i$ en la fase líquida.

$$
\begin{gathered}
G_{i j}=\exp \left(-c_{i j} \tau_{i j}\right) \\
\tau_{i j}=a_{i j}+\frac{b_{i j}}{T}=\frac{\Delta g_{i j}}{R T} \\
a_{i i} b_{i i} c_{i i}=0 T_{i i}=0 G_{i i}=1
\end{gathered}
$$

Considerando el cambio de la energía libre de Gibbs de mezcla $\left(\Delta g_{i j}\right)$ en $\left(\frac{J}{m o l}\right)$, la temperatura $(T)$ en $K$, la constante de los gases ideales $(R)$ en $\left(\frac{J}{m o l K}\right), b_{i j}$ en $K^{-1}$ y los coeficientes $G_{i j}, a_{i j}, \tau_{i j} \mathrm{y}$ $c_{i i}$, adimensionales, los parámetros de interacción binaria para el modelo, son:

Tabla 1. Coeficientes $a_{i j}$ para el modelo de NRTL [13].

\begin{tabular}{c|c|c|c|c}
\hline $\mathbf{i}$ & Butanol & $\begin{array}{c}\mathbf{j} \\
\text { Agua }\end{array}$ & Acetona & Etanol \\
\hline Butanol & 0 & -2.0405 & 10.2979 & 0 \\
\hline Agua & 213.1102 & 0 & 0.0544 & 3.4578 \\
\hline Acetona & -8.8875 & 6,3981 & 0 & -0.3471 \\
\hline Etanol & 0 & -0.8009 & -1.0787 & 0 \\
\hline
\end{tabular}


Tabla 2. Coeficientes $\mathrm{b}_{\mathrm{ij}}$ para el modelo de NRTL [13].

\begin{tabular}{c|c|c|c|c}
\hline $\mathbf{i}$ & Butanol & $\begin{array}{c}\mathbf{j} \\
\text { Agua }\end{array}$ & Acetona & Etanol \\
\hline Butanol & 0 & 763.8692 & $-3,326.5381$ & 128.5015 \\
\hline Agua & $-3,338.9536$ & 0 & 419.9716 & -586.0809 \\
\hline Acetona & $3,077.281$ & $-1,808.991$ & 0 & 206.5973 \\
\hline Etanol & -85.2188 & 246.18 & 479.05 & 0 \\
\hline
\end{tabular}

Tabla 3. Coeficientes $c_{i j}$ para el modelo de NRTL[13].

\begin{tabular}{c|c|c|c|c}
\hline $\mathbf{i}$ & Butanol & $\begin{array}{c}\mathbf{j} \\
\text { Agua }\end{array}$ & Acetona & Etanol \\
\hline Butanol & 0 & 0.3 & 0.3 & 0.3 \\
\hline Agua & 0.3 & 0 & 0.3 & 0.3 \\
\hline Acetona & 0.3 & 0.3 & 0 & 0.3 \\
\hline Etanol & 0.3 & 0.3 & 0.3 & 0 \\
\hline
\end{tabular}

\subsection{Ecuaciones del Virial}

La ecuación del virial nace directamente de la mecánica estadística, considerando en cuenta las fuerzas intermoleculares. Con este modelo se describe el comportamiento de un gas real a partir de una serie de potencias que describen magnitudes molares. En contraste, para los líquidos se utilizó el producto entre la presión y el volumen (PV). En el caso de los gases es más constante el producto PV que la fracción molar, debido a que esta varía con el cambio de presión ejercida en el sistema según la ley de las presiones parciales de Dalton, la cuál considera que la fracción molar es directamente proporcional a su presión parcial, lo que hace a la presión parcial una medida de concentración para los gases que se comportan idealmente, y se encuentren en condiciones de baja a moderadas presiones (menos de $10 \mathrm{~atm}$ ).

Las ecuaciones utilizadas fueron las siguientes [14]:

$$
\begin{gathered}
T_{C i j}=\sqrt{T_{C i}+T_{C j}} \\
Z_{C i j}=\frac{Z_{C i}+Z_{C j}}{2} \\
W_{i j}=\frac{W_{i}+W_{j}}{2}
\end{gathered}
$$

$$
\begin{aligned}
& V_{C i j}=\left(\frac{V_{C i}^{1 / 2}+V_{C j}^{1 / 2}}{2}\right)^{3} \\
& P_{C i j}=\frac{R * T_{C i j}+Z_{C j j}}{V_{C i j}} \\
& T_{r i j}=\frac{T}{T_{C i j}} \\
& b_{0 i j}=0.0833-\frac{0.422}{\left(T_{r i j}\right)^{1.6}} \\
& \grave{B_{\imath \jmath}}=0.139-\frac{0.172}{\left(T_{r i j}\right)^{4.2}} \\
& B_{i j}=\frac{R * T_{C i j}}{P_{C i j}}\left(b_{0 i j}+B_{i j}+W_{i j}\right) \\
& \delta_{i j}=2\left(B_{i j}+B_{i i}+B_{j j}\right) \\
& \operatorname{Ln} \theta_{k}=\frac{P}{R T}\left(B_{K K}+\frac{1}{2} \sum_{i=1}^{n} \sum_{j=1}^{m}\left(2 \delta_{i k}-\delta_{i j}\right)\right) \\
& \varphi^{S a t}{ }_{i}=\exp \left(\frac{P_{r}{ }^{s a t}}{\operatorname{Tr}}\left(\grave{B}_{i i} * W_{i i}+b_{0 i i}\right)\right)
\end{aligned}
$$




$$
\varphi_{i}=\frac{\theta_{i}}{\varphi^{S a t}}
$$

Donde $\varphi_{i}$ es la fugacidad del componente $i$.

$$
\ln P=a-\frac{b}{C+T}
$$

Donde la temperatura está expresada en Kelvin (K) y la presión en $\mathrm{kPa}$.

Donde los parámetros para el modelo se muestran en la tabla 4:

\begin{tabular}{|c|c|c|c|c|c|c|c|c|}
\hline Especie & Te (k) & Pc (bar) & $\begin{array}{c}\text { Vc } \\
\left.\mathbf{c m}^{3} / \mathbf{m o l}\right)\end{array}$ & $\mathrm{Zc}$ & $\mathbf{w}$ & $\mathbf{a}$ & b & $\mathrm{C}$ \\
\hline Butanol & 536.00 & 41.90 & 268.0 & 0.252 & 0.576 & 14.6961 & $2,902.96$ & -102.9116 \\
\hline Agua & 647.60 & 224.02 & 56.0 & 0.229 & 0.344 & 16.53620 & $3,985.440$ & -38.997 \\
\hline Acetona & 508.10 & 47.01 & 209.0 & 0.232 & 0.309 & 14.71710 & $2,975.950$ & -34.523 \\
\hline Etanol & 516.2 & 63 & 167 & 0.248 & 0.635 & 16.1952 & $3,423.53$ & -55.7152 \\
\hline
\end{tabular}

Tabla 4. Constantes Críticas de los gases [15]

Donde Tc es la temperatura critica del componente, $\mathrm{Pc}$ es la presión critica del componente, $\mathrm{Vc}$ es el volumen crítico del componente, $\mathrm{Zc}$ es el factor de compresibilidad crítico del componente, W es el factor acéntrico del componente y a, b, c son los parámetros de cada componente para la ecuación de Antoine.

\subsection{Algoritmo de Punto de Rocío y Punto de Burbuja}

Para realizar el proceso iterativo se utilizó el algoritmo de Rachford y Rice [16], para el cual se fijó una presión $\mathrm{P}$ y se calculó una temperatura $\mathrm{T}$. Lo anterior se puede realizar de dos maneras. En la primera se estima el punto de rocío donde se especifican las fracciones en la fase vapor $\left(y_{i}\right) \mathrm{y}$ se hallan las fracciones en la fase líquida $\left(x_{i}\right) \mathrm{y}$ en la segunda se estima el punto de burbuja, donde se especifican las fracciones en la fase líquida y se hallan las fracciones en la fase vapor [17]. Para el primer caso se debe especificar la presión $\mathrm{P}$ y las fracciones en la fase vapor $Y_{m}$, esto con el fin de estimar una solución inicial utilizando la Ec. (18) para encontrar una temperatura $\mathrm{T}_{0}$ y la Ec.(19) para la fracción en la fase líquida.

$$
\begin{gathered}
T_{0}=\sum_{m} y_{m} T_{m}^{s a t} \\
x_{m}=\frac{y_{m P}}{P_{m}^{s a t}}
\end{gathered}
$$

Donde $T_{0}$ es nuestra temperatura inicial para el proceso de iteración, $T_{m}^{\text {sat }}$ es la temperatura de saturación para cada compuesto a una presión $\mathrm{P}, P_{m}^{\text {sat }}$ es la presión de saturación estimada a una temperatura $T_{0}$ y $X_{m}$ es la fracción en la fase líquida. Debido a que es una solución estimada, la sumatoria de las fracciones en la fase líquida no es exactamente igual a la unidad y por tanto se debe realizar un proceso de normalización con la Ec. (20).

$$
\overline{X_{m}}=\frac{x_{m}}{\sum_{m} x_{m}}
$$

Una vez obtenida la solución estimada, se calculan el coeficiente de fugacidad para la fase vapor $\left(\varphi_{m}\right)$ y el coeficiente de actividad para la fase líquida $\left(\gamma_{m}\right)$, con el fin de calcular el coeficiente de distribución $\left(K_{m}\right)$ (Ec. (21)) y unas nuevas fracciones corregidas en la fase líquida (Ec. (22) y (23)).

$$
\begin{gathered}
K_{m}=\frac{\gamma_{m}\left(T_{0}, P,\left\{\overline{X_{m}}\right\}\right)}{\varphi_{m}\left(T_{0}, P,\left\{y_{m}\right\}\right)} \\
x_{m, 1}=\frac{y_{m}}{K_{m}} \\
\overline{X_{m, 1}}=\frac{X_{m, 1}}{\sum_{m} X_{m, 1}}
\end{gathered}
$$

Al obtener $\overline{X_{m, 1}}$ y $\overline{X_{m}}$ podemos calcular un error $1(e 1)$ correspondiente a la fracción en la fase líquida (Ec. (24)). La tolerancia para este error fue tomada como $e 1 \leq 1 * 10^{-6}$. 


$$
e 1 \leq \sum_{m}\left|\overline{X_{m, 1}}-\overline{X_{m}}\right|
$$

Una vez calculado el error se procede a asignar el valor de $\overline{X_{m, 1}}$ a $\overline{X_{m}}$. En el caso de que el error no cumpla el condicional del error (Ec. (24)); procedemos a calcular nuevamente los coeficientes de distribución hasta que el error sea menor al valor fijado. Una vez terminado este proceso iterativo para las fracciones de la fase líquida, se comienza el proceso para hallar la temperatura correcta, iniciando con el cálculo de una función error para la temperatura, la cual viene dada en la Ec. (25), y el valor absoluto de esta función será el error $2(e 2)$. Para este caso se fijó un error de $e 2 \leq 1 * 10^{-6}$.

$$
\begin{gathered}
f\left(T_{0}\right)=1-\sum \frac{Y_{m}}{K_{m}\left(T_{0}, P,\left\{\overline{X_{m}}\right\},\left\{Y_{m}\right\}\right)} \\
e 2 \leq\left|f\left(T_{0}\right)\right|
\end{gathered}
$$

Si el error se cumple (Ec. (26)), la iteración ha terminado, en caso contrario se procede a calcular una nueva temperatura. Para el cálculo de esta nueva temperatura, se debe derivar la función error de la temperatura $\left(f^{\prime}\left(T_{0}\right)\right.$. Para simplificar el proceso de derivación se utilizó un método numérico (derivación numérica por diferencias centradas), el cual consiste en calcular la función error con un cambio de temperatura $(\Delta T)$, como se muestra en la Ec. (27) (se aconseja utilizar un $\Delta T$ pequeño para que la solución sea más exacta) [18].

$$
f^{\prime}\left(T_{0}\right)=\frac{f\left(T_{0}+\Delta T\right)-f\left(T_{0}-\Delta t\right)}{2 \Delta T}
$$

El cálculo de la nueva temperatura $T_{1}$ viene dada por la Ec. (27).

$$
T_{1}=T_{0}-\frac{f\left(T_{0}\right)}{f^{\prime}\left(T_{0}\right)}
$$

Una vez calculada $T_{1}$ le asignamos el valor de $T_{0}$ y una vez realizado esto, calculamos nuevamente los coeficientes de distribución para el cálculo de las fracciones en la fase líquida. Se realiza este proceso iterativo hasta cumplir que $e 2$ sea menor al estipulado.
Para el caso del cálculo del punto de burbuja, se debe especificar la presión $\mathrm{P}$ y las fracciones en la fase líquida $x_{m}$. Con el fin de calcular una temperatura inicial de iteración, se utilizó la Ec. (29) para encontrar una temperatura $T_{0}$ y la Ec. (30) para la fracción en la fase vapor.

$$
\begin{gathered}
T_{0}=\sum_{m} x_{m} T_{m}^{\text {sat }} \\
y_{m}=\frac{x_{m} P_{m}^{\text {sat }}}{P}
\end{gathered}
$$

Al igual que en el caso anterior se debe normalizar las fracciones $y_{m}$ y al realizar el cálculo de $K_{m}$, en este caso debemos utilizar la Ec. (31) para hallar $y_{m, 1}$, las cuales deben ser normalizadas.

$$
y_{m, 1}=x_{m} K_{m}
$$

Se calcula el $e 1$, pero en este caso vendrá dado por:

$$
\left|\overline{Y_{m, 1}}-\overline{Y_{m}}\right| \leq e 1
$$

Y se realiza el proceso iterativo hasta cumplir esta condición. Para el caso del punto de burbuja se utilizó la siguiente función objetivo (Ec. (33)).

$f\left(T_{0}\right)=1-\sum x_{m} K_{m}\left(T_{0}, P,\left\{\overline{Y_{m}}\right\},\left\{x_{m}\right\}\right)$

Una vez calculada la función error utilizamos la Ec. (27) para calcular la nueva temperatura y poder continuar con el proceso iterativo al igual que en el caso anterior.

\section{RESULTADOS}

El análisis se centra en el comportamiento del azeótropo entre el agua y el butanol, ya que este último es el compuesto de interés, y se separa fácilmente del etanol y la acetona utilizando una destilación simple. Por tanto, se realizó primero simulaciones a una presión de $100 \mathrm{kPa}$, para observar el comportamiento de este a una presión cercana a la atmosférica. Como se puede ver en las figuras 1 y 2, el solapamiento de la gráfica XY entre el butanol y el agua nos revela la existencia del azeótropo a presión atmosférica. También se 
puede apreciar la reducción del solapamiento con la disminución de la concentración del etanol, como se aprecia en la figura 1 el solapamiento entre el agua y el butanol se inicia alrededor de 0,22 en su fracción liquida y en la figura 2 este inicia aproximadamente en 0,05.

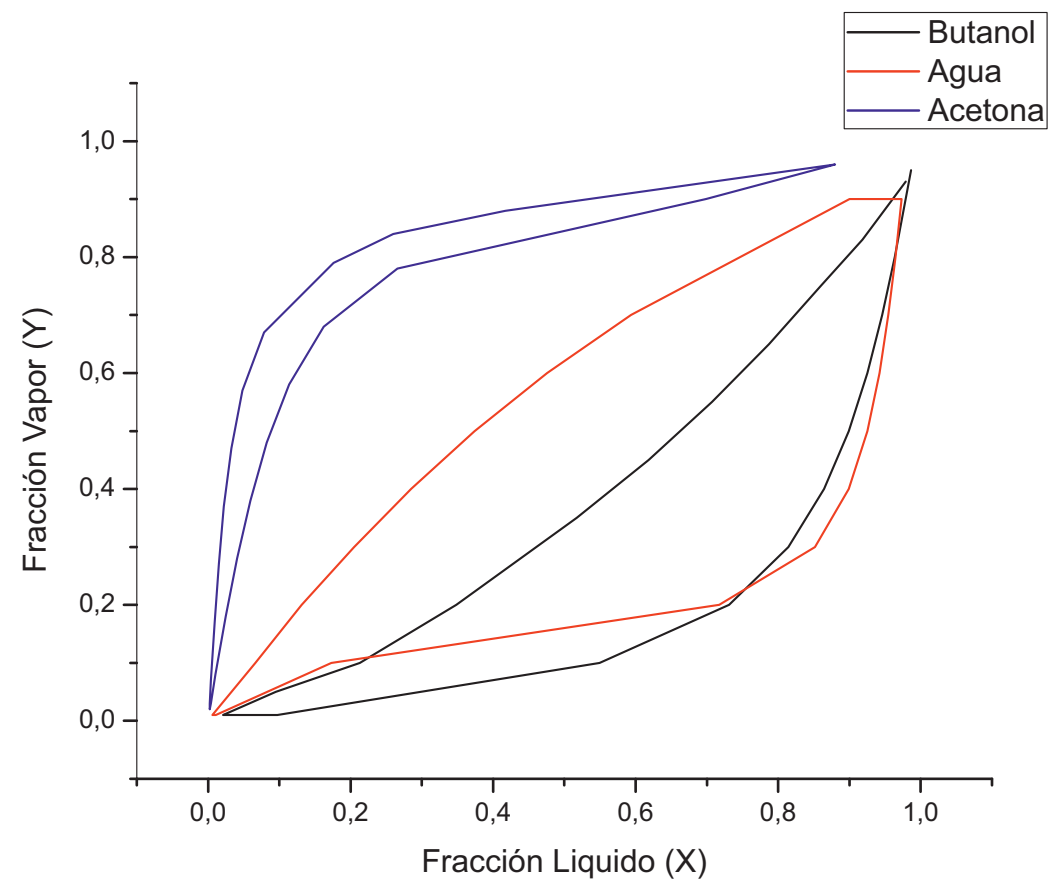

Figura 1. Simulación a $100 \mathrm{kPa}$ y a $\mathrm{YC}_{2} \mathrm{H}_{6} \mathrm{O}=2 \%$

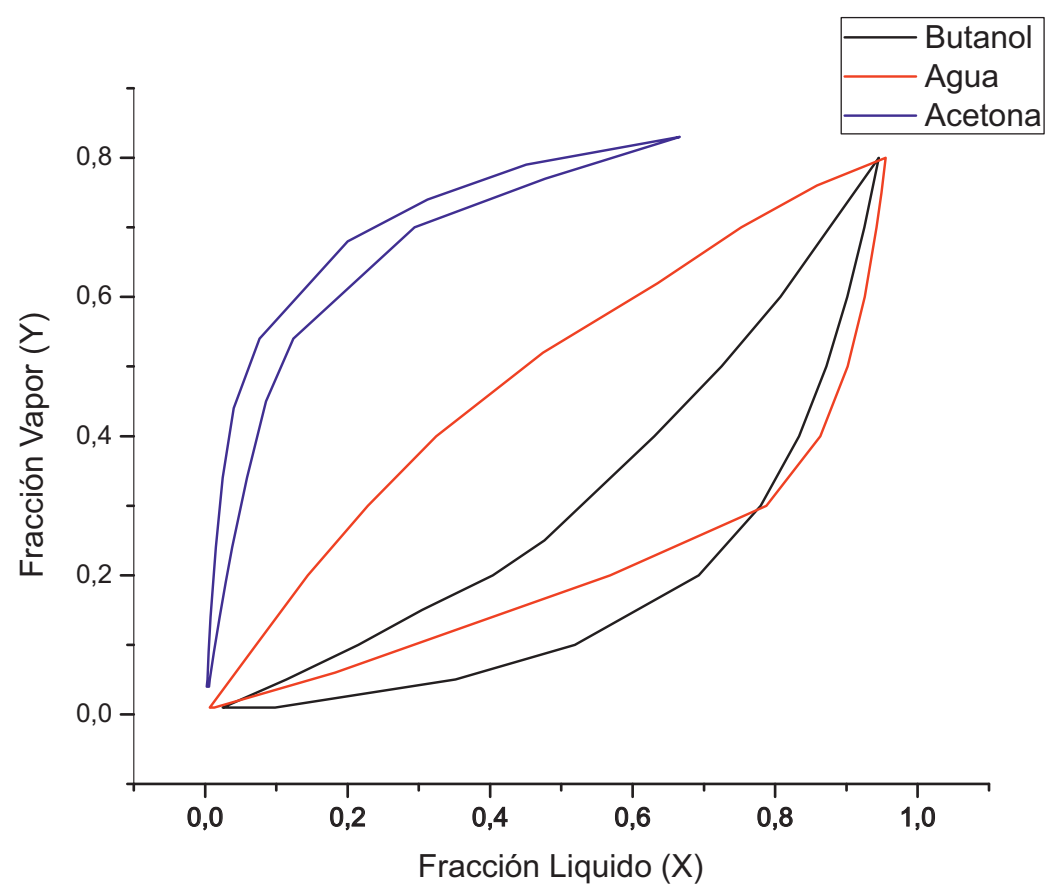

Figura 2. Simulación a $100 \mathrm{kPA}$ y a $\mathrm{YC}_{2} \mathrm{H}_{6} \mathrm{O}=15 \%$

También se puede ver que la fase vapor está compuesta principalmente de acetona, ya que este es el compuesto más volátil de la mezcla. Al reducir la presión se empieza a ver la reducción del azeótropo que se forma entre el agua y el butanol como apreciamos en las figuras 3 y 4 . 


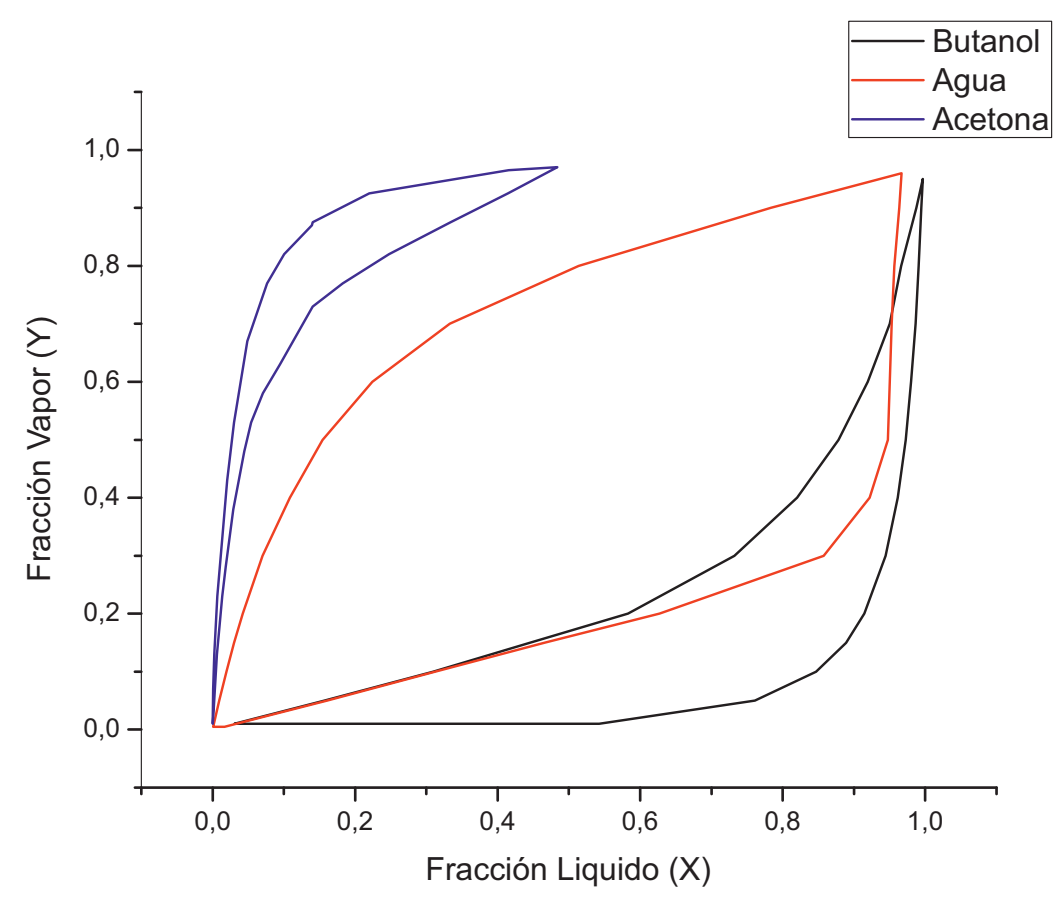

Figura 3. Simulación a $1 \mathrm{kPA}$ y a $\mathrm{YC}_{2} \mathrm{H}_{6} \mathrm{O}=2 \%$

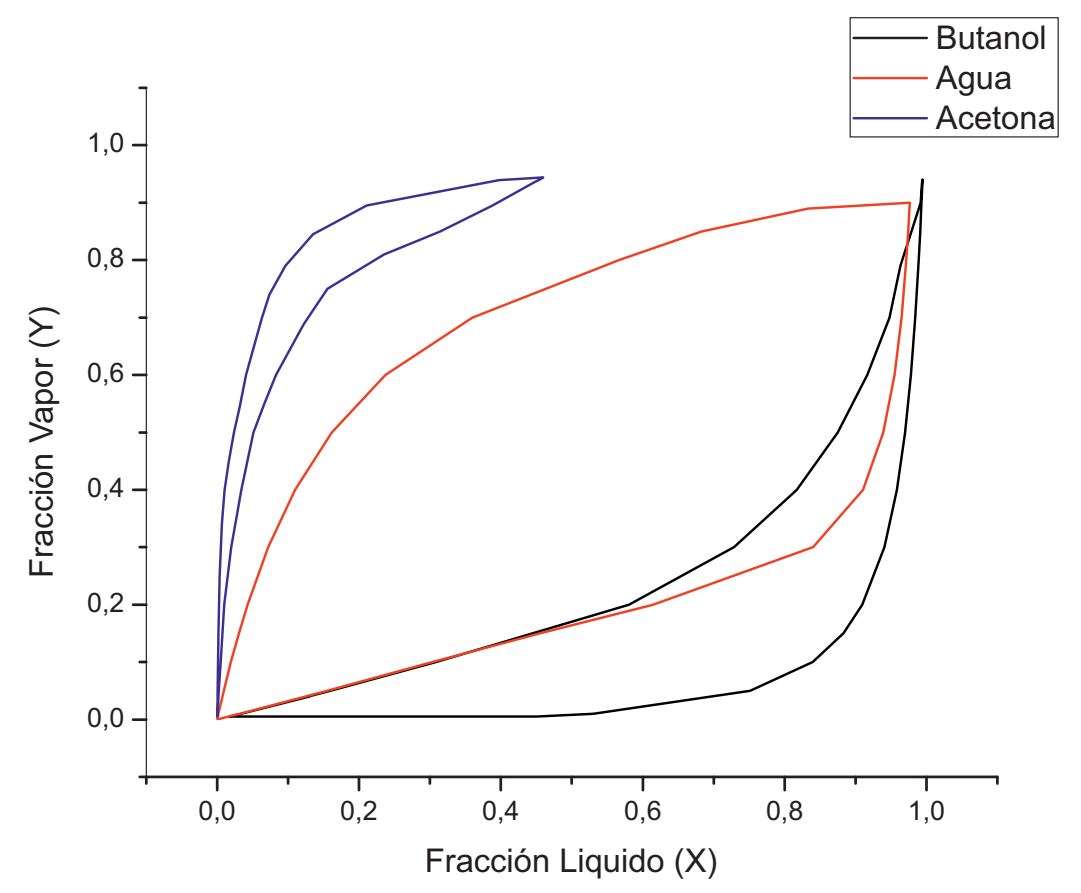

Figura 4. Simulación a $\mathrm{IkPa}$ y a $\mathrm{YC}_{2} \mathrm{H}_{6} \mathrm{O}=5 \%$

Las concentraciones del etanol, $2 \%$ y $5 \%$, siguen favoreciendo el azeotropo entre el agua y el butanol a $1 \mathrm{kPa}$, pero aumentando esta concentración a $10 \%$ y $15 \%$ se puede observar que la interacción con el etanol elimina el azeótropo (figuras 5 y 6). En cuanto a la concentración de acetona en la fase líquida se aprecia la reducción de su fracción molar con relación a la presión de $100 \mathrm{kPa}$, debido a que es el compuesto mas volátil, mientras que la fase líquida empieza a concentrarse con butanol, debido a que el valor de su fugacidad empieza a disminuir hasta valores de 0.85 . 


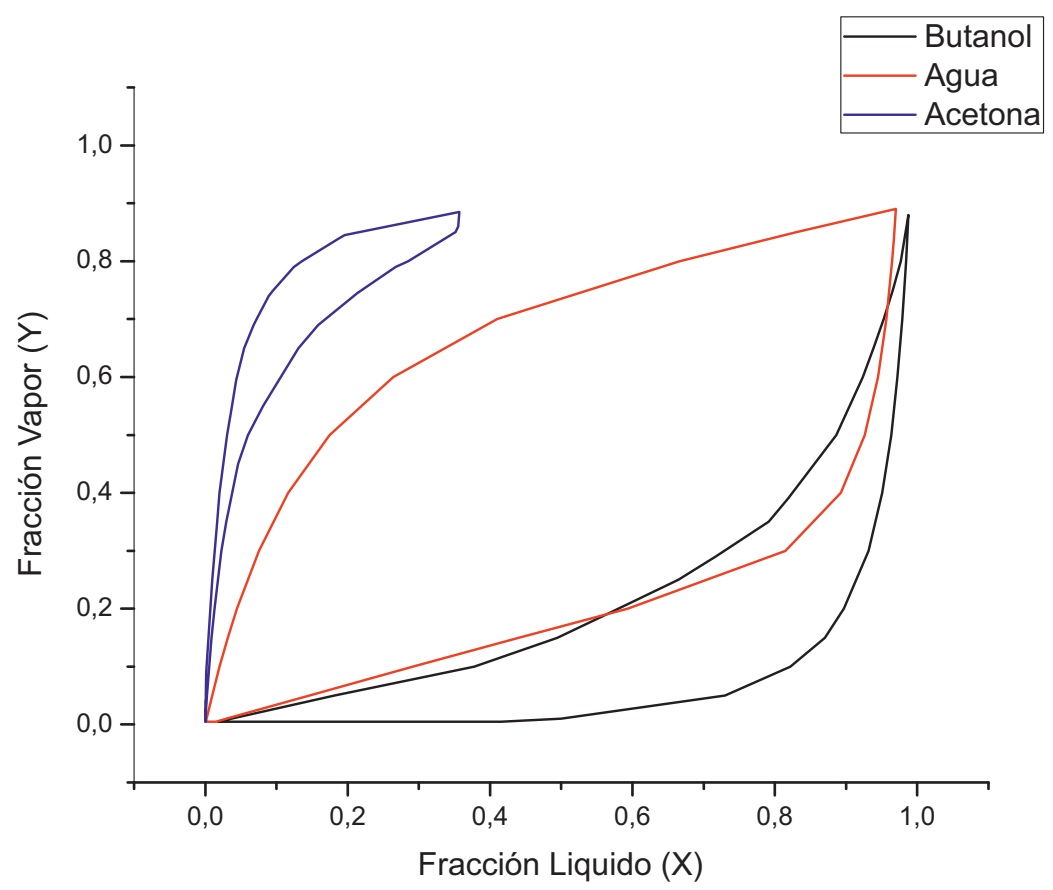

Figura 5. Simulación a $1 \mathrm{kPa}$ y a $\mathrm{YC}_{2} \mathrm{H}_{6} \mathrm{O}=10 \%$

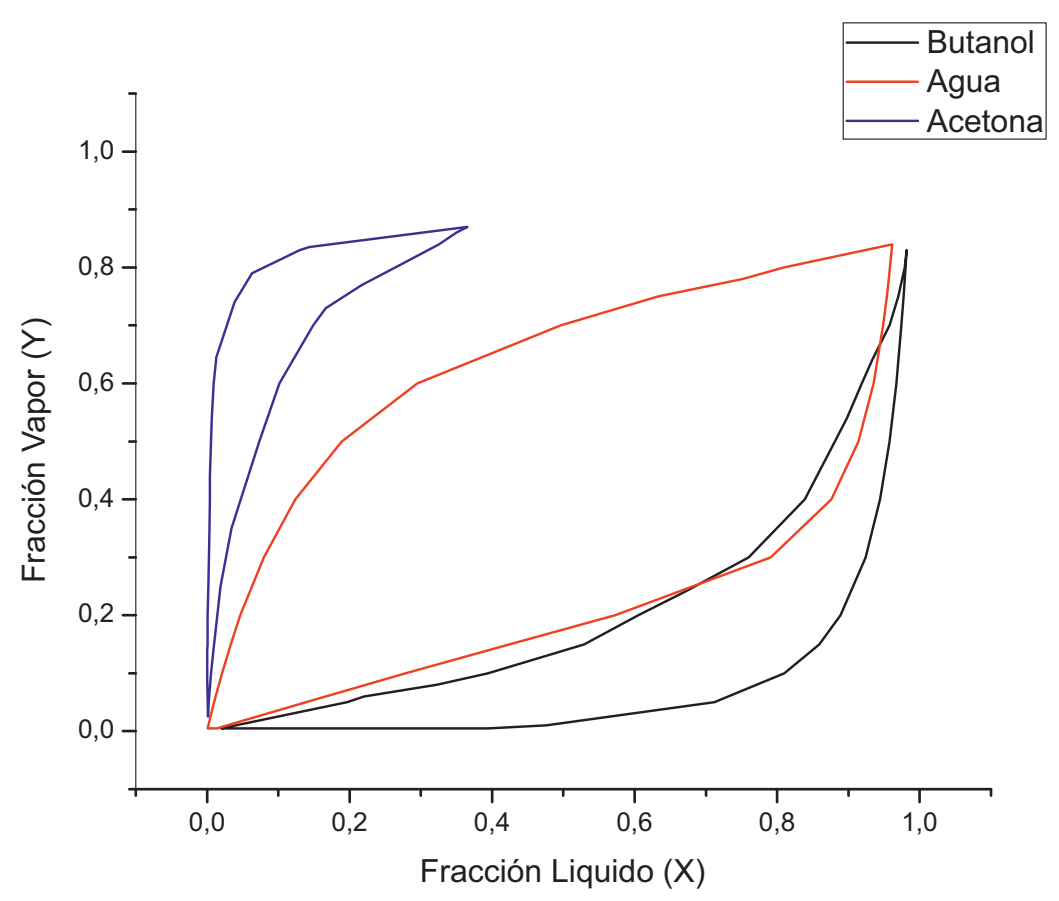

Figura 6. Simulación a $1 \mathrm{kPa}$ y a $\mathrm{YC}_{2} \mathrm{H}_{6} \mathrm{O}=15 \%$

A una presión de $0.5 \mathrm{kPa}$ se observa la eliminación del azeótropo entre el butanol y el agua (ver Figuras 7 y 8). Al aumentar la concentración de etanol, la concentración de butanol en la fase líquida empieza a disminuir, tal como se aprecia en la figura 7 , donde además vemos el aumento de la concentración del agua y del butanol en la fase líquida a diferencia de la figura 6 . 


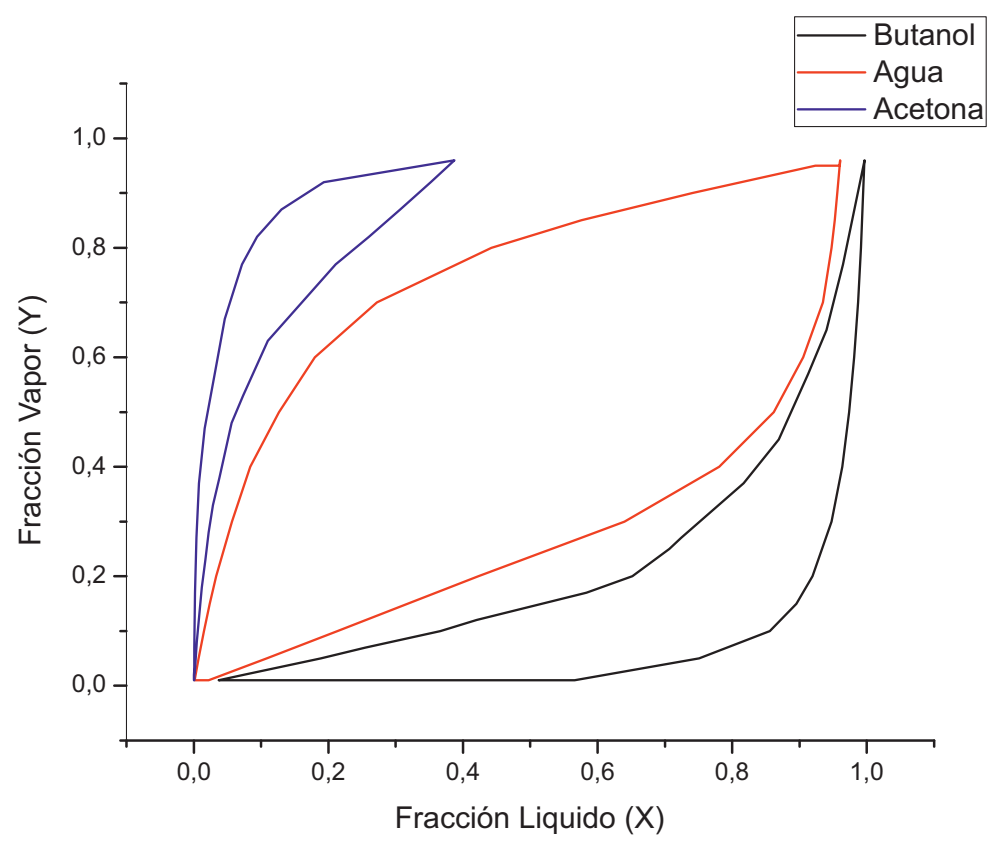

Figura 7. Simulación a $0.5 \mathrm{kPa}$ y a $\mathrm{YC}_{2} \mathrm{H}_{6} \mathrm{O}=2 \%$

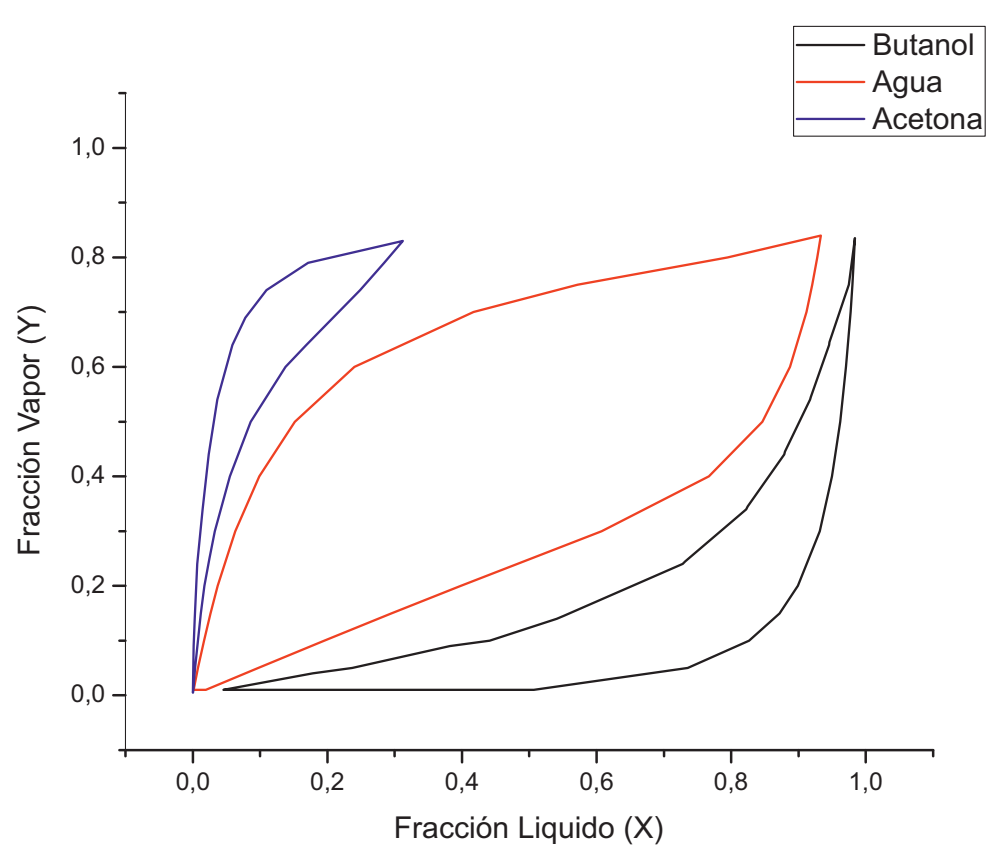

Figura 8. Simulación a $0.5 \mathrm{kPa}$ y a $\mathrm{YC}_{2} \mathrm{H}_{6} \mathrm{O}=15 \%$

El solapamiento que se produce a $0.1 \mathrm{kPa}$ es debido a la formación de algún tipo de interacción entre el agua y la acetona. El comportamiento del diagrama de fase que existe entre el agua y el butanol se aleja evitando la formación de un azeotropo (figura 9), haciendo a esta presión ideal para la separación del butanol y el agua por variación de temperatura. El inconveniente que presenta el aumento de la concentración de butanol en la fase líquida es indeseable en procesos como la pervaporación, pero es deseable para procesos como la destilación, puesto que el butanol reside en la corriente de fondo y el agua en la corriente del destilado. De igual manera, al realizar la simulación a $0.5 \mathrm{kPa}$ la fracción de butanol en la fase líquida empieza a disminuir con el aumento de la concentración de etanol (figura 10), dado que se empieza a reducir la concentración de la acetona tanto en la fase líquida como en la fase de vapor. 


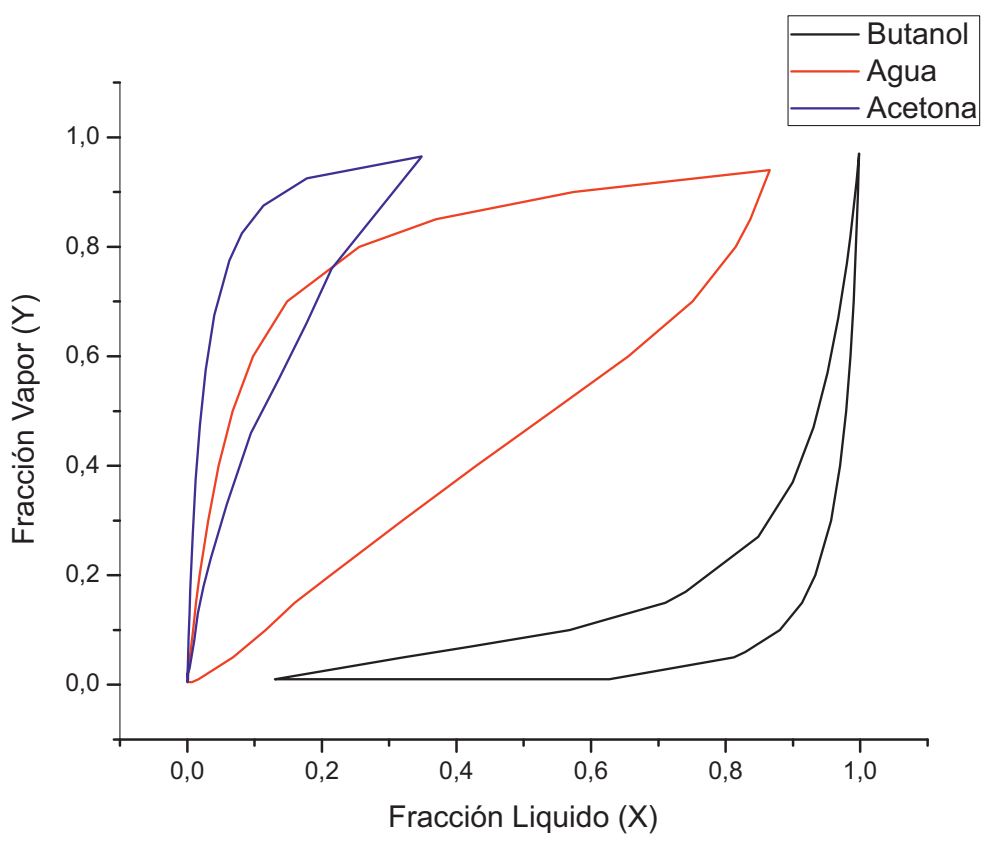

Figura 9. Simulación a $0.1 \mathrm{kPa}$ y a $\mathrm{YC}_{2} \mathrm{H}_{6} \mathrm{O}=2 \%$

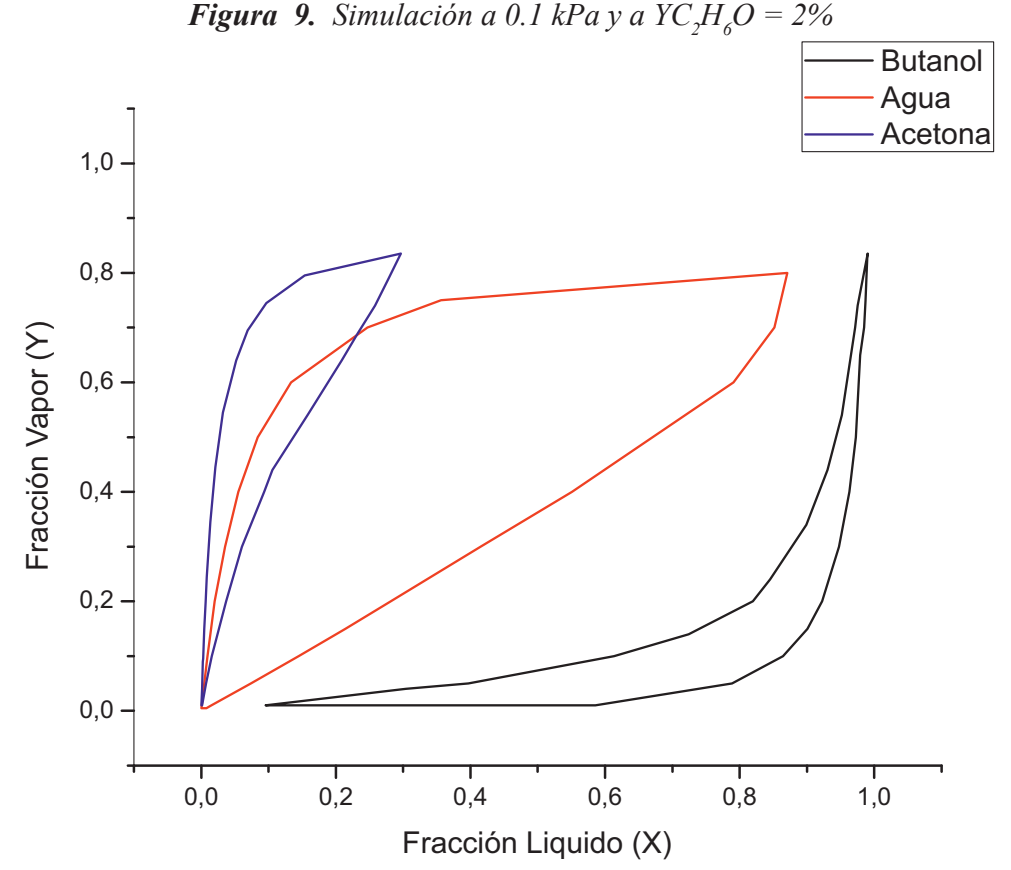

Figura 10. Simulación a $0.1 \mathrm{kPA}$ y a $\mathrm{YC}_{2} \mathrm{H}_{6} \mathrm{O}=15 \%$

Alvariarlaconcentración de etanol a concentración fija de acetona se puede apreciar que a $1 \mathrm{kPa}$ existe un doble azeótropo agua-etanol y aguabutanol (figura 11), el cual también se presenta a presión atmosférica. De igual manera, reduciendo la presión, el azeótropo entre el agua y el butanol se va desapareciendo, aunque en comparación con el caso anterior cuando la presión fue de $0,5 \mathrm{kPa}$ todavía existen pequeños puntos donde prevalece el azeótropo entre el agua y el butanol, debido a la diferencia que se presentó en cuanto al cambio de actividad del agua y el butanol, lo cuál genera la variación de la concentración del etanol (figura 12) a diferencia de la acetona (figura 4). Por último, en la simulación a $0,1 \mathrm{kPa}$ vemos que se elimina el azeótropo butanol-agua (figura 13). En resumen, el azeótropo entre el agua y el etanol no se eliminó con el cambio de presión debido a que se generaron interacciones de ambos componentes con el butanol y la acetona; lo cual modificó sus actividades con respecto a aquellas que presentan en estado puro. 
Jairo Antonio Cubillos Lobo et. al.

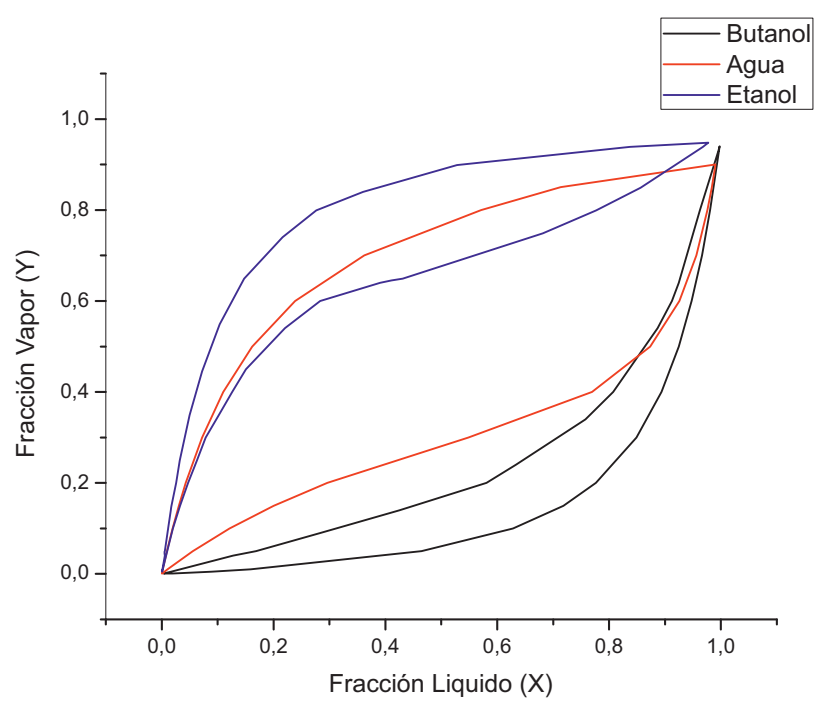

Figura 11. Simulación a $1 \mathrm{kPA}$ y a $\mathrm{YC}_{3} \mathrm{H}_{6} \mathrm{O}=5 \%$

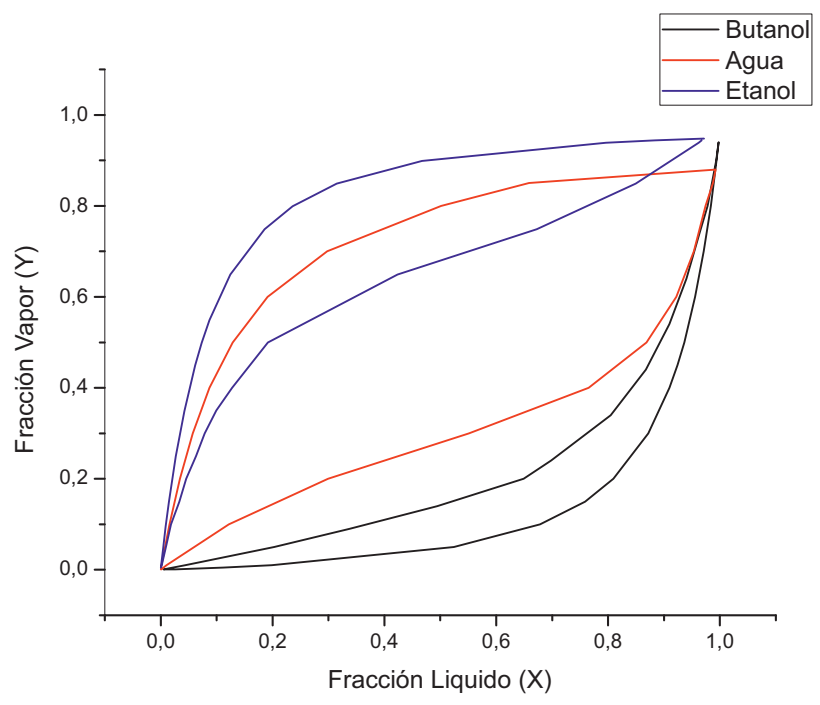

Figura 12. Simulación a $0.5 \mathrm{kPa}$ y a $\mathrm{YC}_{3} \mathrm{H}_{6} \mathrm{O}=5 \%$

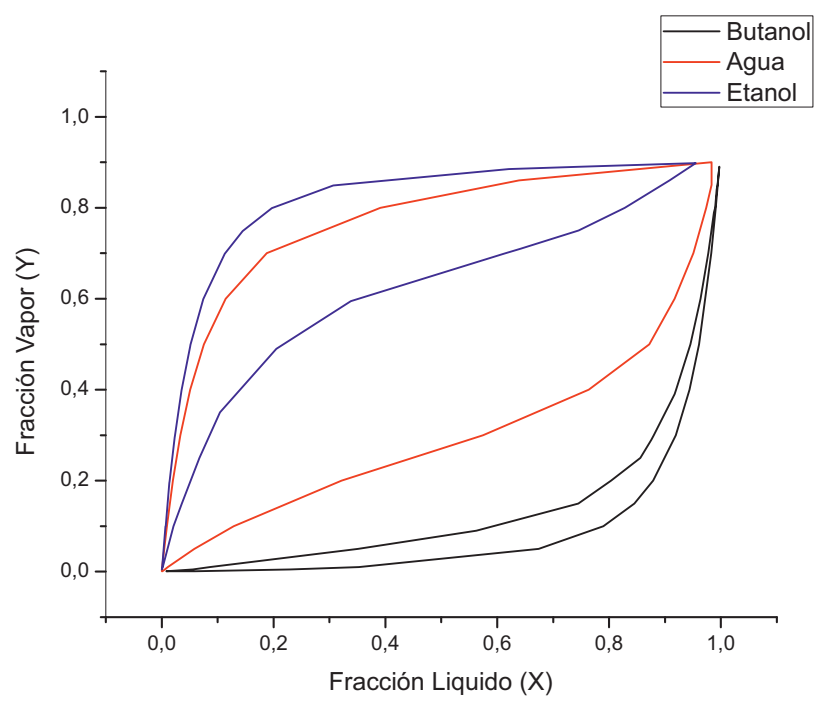

Figura 13. Simulación a $0.1 \mathrm{kPA}$ y a $\mathrm{YC}_{3} \mathrm{H}_{6} \mathrm{O}=10 \%$ 
La variación de la actividad es un factor clave para el cálculo y la explicación a los cambios en las formas de las curvas de equilibrio L-V. Se encontraron valores entre 0.2 y 9 para acetona, 1 y 4 para etanol, 1 y 3 para el agua, y desde 0.6 hasta 2 para el butanol. Las actividades cambian su valor, especialmente cuando la concentración del componente se acerca a su dilución infinita y cuando la presión disminuye, experimentando un aumento con este parámetro.

Por tener los valores de actividad más altos, la acetona es el componente que más interactua con otros compuestos de la mezcla y tiene un comportamiento menos estable a diferencia del butanol que presentó los valores más bajos de actividad. La más baja actividad del etanol con respecto a la acetona indica la poca variación de las gráficas XY (figura 11, 12 y 13) cuando la concentración de la acetona se mantuvo constante y la concentración del etanol se variaba.

La fugacidad mantuvo valores en un rango pequeño de 0.8 a 1 para todos los componentes, debido a que las presiones utilizadas fueron bajas y por eso la desviación con respecto a la curva ideal fue pequeña. Para las simulaciones a 100 $\mathrm{kPa}$ y $1 \mathrm{kPa}$ la fugacidad se mantuvo entre $0.9 \mathrm{y}$ 1, a excepción del butanol que presentó valores entre 0.86 y 1 , valores que se explican debido a que es el componente menos volátil de la mezcla.

El coeficiente de distribución () es la relación que existe entre el coeficiente de actividad y de fugacidad de un componente en mezcla (ecuación 21). Para este parámetro, los valores mas bajos corresponden al butanol, debido a que es el componente menos volátil de la mezcla, con valores que van desde 0.8 hasta 2.2. Por otro lado, los valores más altos se presentaron con acetona alcanzando valores entre 0,2 y 10. En el caso del agua se encontraron valores entre 0.95 y 2.5 y para el etanol entre 1 y 3.7. Estos valores están marcadamente influenciados por la actividad del componente debido a que los valores obtenidos por la fugacidad son valores cercanos a 1 .

\section{CONCLUSIONES}

En este trabajo se realizó una simulación del equilibrio líquido-vapor de 4 componentes (agua, acetona, butanol y etanol) variando las presiones, con el propósito de evaluar el comportamiento del azeótropo entre el agua y el butanol. Se confirmó que a presiones cercana a la atmosférica $(100 \mathrm{kPa})$ ocurre el azeótropo, pero al reducir la presión por debajo de $0.5 \mathrm{kPa}$ este empieza a desaparecer, lo que hace posible la aplicación de una destilación simple a bajas presiones para la recuperación del butanol a partir de una mezcla de fermentación ABE. También se estableció que el aumento en la concentración de etanol favoreció la reducción del azeótropo agua-butanol y la concentración de butanol en la fase de vapor.

Al reducir significativamente la presión, la concentración de butanol en la fase líquida aumenta notoriamente, lo que no es favorable a procesos como la pervaporación, debido a que reduce la posibilidad de vaporización instantánea del butanol al hacer contacto con la membrana que se utiliza en este proceso, pero favorece procesos como la destilación simple al enriquecer la fase liquida con butanol y la fase vapor con agua; otro factor que afecta la concentración del butanol en la fase líquida es la concentración del etanol en la fase vapor, ya que es directamente proporcional a la fracción de butanol en el líquido.

La condición que afectó más la actividad de los componentes (acetona, agua, butanol y etanol) fue la variación de la concentración de estos, aunque en menor medida también intervinieron otros factores como la presión y la temperatura. $\mathrm{La}$ acetona es el componente de más interacción en la solución ya que presenta los valores de actividad más altos (hasta 9). Al mantener presiones bajas los componentes en fase vapor se comportan como gases ideales, lo cual se confirmó con sus valores de fugacidad cercanos a 1 .

\section{BIBLIOGRAFÍA}

[1] P.H. Pfromm, V. Amanor-Boadu, R. Nelson, P. Vadlani, R. Madl, "Bio-butanol vs. bio-ethanol: A technical and economic assessment for corn and switchgrass fermented by yeast or Clostridium acetobutylicum", Biomass Bioenerg, vol. 34, pp. 515-524, 2010. 10.1016/j. biombioe.2009.12.017.

[2] J.A. Moreno, J.A. Cubillos, "Biobutanol Como Combustible: Una Alternativa 
Alternativa Sustentable", Investigación Joven, vol.4, pp. 45-50, 2017

[3] C. Jin, M. Yao, H. Liu, C.F.F. Lee, J. Ji, "Progress in the production and application of n-butanol as a biofuel", Renew. Sustain. Energ. Rev., vol. 15, pp. 4080-4106. 2011. 10.1016/j.rser.2011.06.001.

[4] J. Zhang, L. Yu, M. Lin, Q. Yan, S.T. Yang, "n-Butanol production from sucrose and sugarcane juice by engineered Clostridium tyrobutyricum overexpressing sucrose catabolism genes and adhE2", Bioresour. Technol., vol. 233, pp. 51-57, 2017. 10.1016/j.biortech.2017.02.079.

[5] X. Kong, A. He, J. Zhao, H. Wu, J. Ma, C. Wei, W. Jin, M. Jiang, "Efficient acetonebutanol-ethanol (ABE) production by a butanol-tolerant mutant of Clostridium beijerinckii in a fermentation-pervaporation coupled process", Biochem. Eng. J., vol. 105, pp. 90-96, 2016. 10.1016/j. bej.2015.09.013.

[6] N. Abdehagh, F.H. Tezel, J. Thibault, "Separation techniques in butanol production: Challenges and developments", Biomass Bioenerg., vol. 60, pp. 222-246, 2014. 10.1016/j.biombioe.2013.10.003.

[7] I. Patraşcu, C.S. Bîldea, A.A. Kiss, "Ecoefficient butanol separation in the ABE fermentation process", Sep. Purif. Technol., vol. 177, pp. 49-61, 2017. 10.1016/j. seppur.2016.12.008.

[8] J.A. Cubillos, F. Bustamante, A. Acosta, "Modelación y simulación de un pervaporador acoplado a un proceso de sacarificación-fermentación para la producción de etanol", Rev. Fac. Ing. vol. 24, pp. 51-68, 2015. 10.19053/01211129.3848.

[9] R.H. Perry, "Perry's Chemical Engineers' Handbook", McGraw-Hill: New York, 1997, tomo IV, sección 17.
[10] G. Dalle Ave, T.A. Adams, "Technoeconomic comparison of Acetone-ButanolEthanol fermentation using various extractants", Energy Conv. Manag., vol. 156, pp. 288-300, 2018. 10.1016/j. enconman.2017.11.020.

[11] E.A. Crespo, L.P. Silva, M.A.R. Martins, L. Fernandez, J. Ortega, O. Ferreira, G. Sadowski, C. Held, S.P. Pinho, J.A.P. Coutinho, "Characterization and Modeling of the Liquid Phase of Deep Eutectic Solvents Based on Fatty Acids/Alcohols and Choline Chloride", Ind. Eng. Chem. Res., vol. 56, pp. 12192-12202, 2017. 10.1021/acs.iecr.7b02382.

[12] E.J. Henley, J.D. Seader, "EquilibriumStage Separation Operations in Chemical Engineering”, John Wiley \& Sons, New York, 1981, pp. 212-215.

[13] W. Kaminski, A. Górak, A. Kubiczek, "Modeling of liquid-liquid equilibrium in the quinary system of water, acetone, n-butanol, ethanol, and ionic liquid", Fluid Phase Equilib., vol. 384, pp. 114-121, 2014. 10.1016/j.fluid.2014.10.017.

[14] J.P.M. Trusler, "The virial equation of state", Exp. Thermodyn.,vol. 5, pp. 35-74, 2000. 10.1016/S1874-5644(00)80014-4.

[15] G. Reklaitis, "Balance De Materia Y Energia”, Interameri, Mexico, D.F., 1986. Pp 619-622.

[16] H.B. Callen, "Thermodynamics and an Introduction to Thermostatistics", John Wiley \& Sons, New York, 1995, pp. 11-212

[17] B.E. Poling, J.M. Prausnitz, J.P. O’Connell, "The properties of gases \& liquids", McGraw-Hill, 1988. Pp 8.1-8.204.

[18] S.C. Chapra, R.P. Canale, "Métodos Matemáticos Para Ingenieros”, McGrawHill, 2006, pp. 90-95. 